\title{
Kn 26: a textbook case hourglass planetary nebula with a twist
}

\author{
Martín A. Guerrero ${ }^{1}$, Roberto Vázquez ${ }^{2}$, Luis F. Miranda ${ }^{3,4}$ \\ and Gerardo Ramos-Larios ${ }^{5}$ \\ ${ }^{1}$ Instituto de Astrofísica de Andalucía, IAA-CSIC, Spain \\ email: mar@iaa.es \\ ${ }^{2}$ Instituto de Astronomía, Universidad Nacional Autónoma de México, Ensenada, Mexico \\ email: vazquez@astro.unam.mx \\ ${ }^{3}$ Departamento de Fisica Aplicada, Universidade de Vigo, Vigo, Spain \\ ${ }^{4}$ Consejo Superior de Investigaciones Científicas, Madrid, Spain \\ email: lfm@iaa.es \\ ${ }^{5}$ Instituto de Astronomía y Meteorología, Jalisco, Mexico \\ email: gerardo@astro.iam.udg.mx
}

\begin{abstract}
Kn 26 is a recently identified planetary nebula (PN). To confirm its nature and study its spatio-kinematical structure, we have obtained high spatial-resolution optical and nearIR narrowband images, high-dispersion long-slit echelle spectra, and intermediate-resolution spectroscopic observations. The new data confirm the PN nature of Kn 26 and reveal features typical of bipolar PNe: hourglass morphology, $\mathrm{H}_{2}$ emission, and nitrogen enrichment. A detailed analysis of its morphology and kinematics, however, suggests the presence of one additional pair of bipolar lobes that would make Kn 26 a new member of the class of quadrupolar PNe.
\end{abstract}

Keywords. planetary nebulae: individual (Kn 26), infrared: ISM

\section{Introduction}

New surveys for PNe are incessantly increasing the number of known PNe in our Galaxy. Using existing digital sky surveys, Jacoby et al. (2010) presented Kn 26, a bipolar PN candidate previously known as the emission line source Lan 384 (Lanning \& Meakes 2000; Eracleous et al. 2002). Here we introduce and describe high spatialresolution optical and near-IR narrowband images of this nebula in conjunction with high- and intermediate-dispersion long-slit spectroscopic observations. A detailed analysis and discussion of these observations will be presented elsewhere (Guerrero et al., in preparation).

\section{Observational Results}

Kn 26 has been observed under sub-arcsec conditions through narrowband optical $\mathrm{H} \alpha$, [N II] and [O III] filters using the Alhambra Faint Object Spectrograph and Camera (ALFOSC) at the $2.56 \mathrm{~m}$ Nordic Optical Telescope (NOT), and near-IR $\mathrm{Br} \gamma, \mathrm{H}_{2}$ and $K$-continuum filters using the Long-slit Intermediate Resolution Infrared Spectrograph (LIRIS) at the $4.2 \mathrm{~m}$ William Herschel Telescope (WHT). The [N II] image shown in the left panel of Figure 1 reveals (a) a central star, (b) a ring-like structure, (c) a pair of bipolar lobes extending $\approx 90^{\prime \prime}$ along P.A. $120^{\circ}$, and $(d)$ a second pair of smaller bipolar lobes extending $35^{\prime \prime}$ along P.A. $55^{\circ}$. The central ring and the bipolar lobes are especially prominent in [N II] and $\mathrm{H}_{2}$, while the innermost regions show higher excitation. The ring-like structure is most likely caused by the projection of the bipolar lobes. 

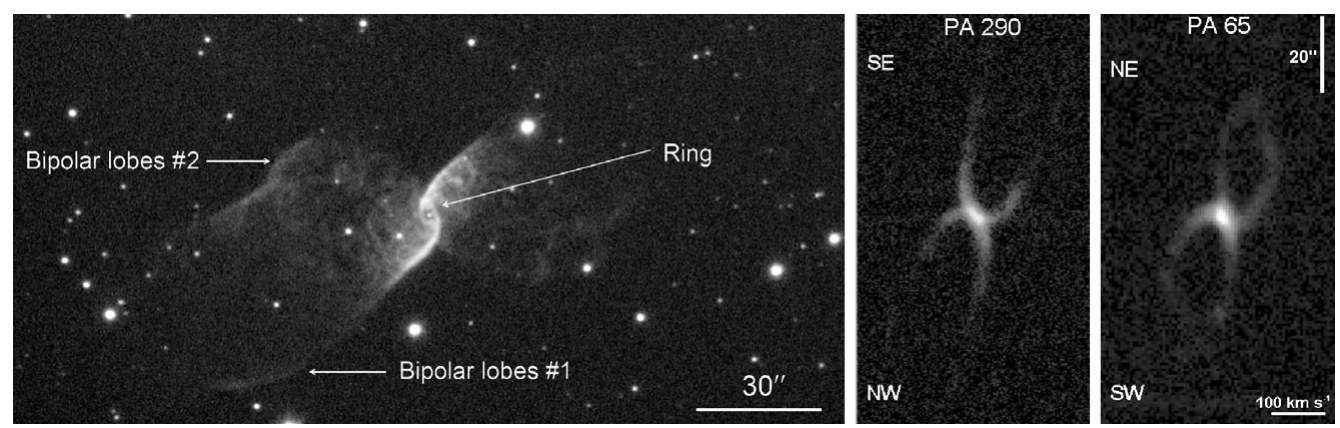

Figure 1. (left) [N II] narrowband image of Kn 26, and (right) position-velocity maps in the same emission line along the two pairs of bipolar lobes at P.A.'s $65^{\circ}$ and $290^{\circ}$. The different morphological components have been labeled on the image.

The intermediate-dispersion optical spectroscopy of Kn 26 consists of a single long-slit observation obtained using the Calar Alto Faint Object Spectrograph (CAFOS) on the $2.2 \mathrm{~m}$ CAHA telescope. The $4^{\prime \prime}$ wide slit was set across the central star and oriented along the east-west direction. The spectrum of Kn 26 includes multiple oxygen, nitrogen and sulfur forbidden lines, as well as hydrogen and helium recombination lines in agreement with previous observations (Eracleous et al. 2002) A preliminary analysis yields values of [O III] $\lambda 5007 / \mathrm{H} \beta \approx 6.4$ and $\mathrm{He}$ II $\lambda 4686 / \mathrm{H} \beta \approx 0.24$ typical of $\mathrm{PNe}$ rather than $\mathrm{H}$ II regions. The relatively high values of the ratios of $[\mathrm{S} \mathrm{II}] / \mathrm{H} \alpha \approx 0.24$ and $[\mathrm{N} \mathrm{II}] / \mathrm{H} \alpha \approx 1.0$ may imply shock-excitation and nitrogen over-abundance. Finally, the density-sensitive ratio [S II] $\lambda 6716 /[\mathrm{S} \mathrm{II}] \lambda 6731 \approx 1.36$ implies low electron density, $\leqslant 100 \mathrm{~cm}^{-3}$.

Long-slit high-dispersion spectroscopy on the $\mathrm{H} \alpha$ and [N II] $\lambda 6583$ lines of $\mathrm{Kn} 26$ has been acquired using the Manchester Echelle Spectrometer (MES) on the $2.1 \mathrm{~m}$ SPM telescope. The $2^{\prime \prime}$ wide slit was set across the central star and oriented along P.A. $65^{\circ}$ and $290^{\circ}$ with spectral resolutions $\approx 12 \mathrm{~km} \mathrm{~s}^{-1}$ and $\approx 6 \mathrm{~km} \mathrm{~s}^{-1}$, respectively. The positionvelocity maps of the $[\mathrm{N} \mathrm{II}] \lambda 6583$ line (Figure 1-right) clearly reveal bipolar kinematics. Whereas the East (West) lobes recedes (moves away) from us, the two pair of bipolar lobes have different inclinations and expansion velocities.

\section{Discussion and Work in Progress}

Our deep, high-resolution narrowband images of Kn 26 have clearly revealed two pairs of bipolar lobes, thus adding this source to the limited subset of PNe with quadrupolar or multipolar morphology (Manchado et al. 1996; Sahai 2000). The two pairs of bipolar lobes do not share the same symmetry axis, and detailed analysis using SHAPE (Steffen et al. 2011) is in progress. Preliminary analysis of the intermediate-dispersion spectroscopic data indicates low density and high excitation, and suggests signs of shock-excitation and nitrogen overabundances that are typical of bipolar PNe (Corradi \& Schwarz 1995).

\section{References}

Corradi, R. L. M. \& Schwarz, H. E. 1995, A\&A, 293, 871.

Eracleous, M., Wade, R. A., Mateen, M., \& Lanning, H. H. 2002, PASP, 114, 207.

Jacoby, G. H., Kronberger, M., Patchick, D., Teutsch, P., Saloranta, J., Howell, M., Crisp, R., Riddle, D., Acker, A., Frew, D. J., \& Parker, Q. A. 2010, PASA, 27, 156.

Lanning, H. H. \& Meakes, M. 2000, PASP, 112, 251.

Manchado, A., Stanghellini, L., \& Guerrero, M. A. 1996, ApJ, 466, L95

Sahai, R. 2000, ApJ, 537, L43

Steffen, W., Koning, N., Wenger, S., Morisset, C., \& Magnor, M. 2011, IEEE Transactions on Visualization and Computer Graphics, 17, 454 